Original Article

\title{
The Reliability of Rehabilitative Ultrasound Imaging of the Cross-sectional Area of the Lumbar Multifidus Muscles in the PNF Pattern
}

\author{
Qiuchen Huang, PT ${ }^{1-3)^{*}}$, Desheng Li, $\mathrm{PT}^{1-3)}$, Yuying Zhang, $\mathrm{PT}^{1)}$,Anming Hu, $\mathrm{PhD}^{2,3}$ \\ Ming Huo, PT, PhD ${ }^{4}$, Hitoshi Maruyama, PT, $\mathrm{PhD}^{1)}$ \\ 1) Department of Physical Therapy, International University of Health and Welfare: 2600-1 \\ Kitakanemaru, Ohtawara City, Tochigi 324-8501, Japan \\ 2) Department of Physical Therapy, China Rehabilitation Research Center, China \\ 3) School of Rehabilitation Medicine, Capital Medical University, China \\ 4) Himeji Dokkyo University, Japan
}

\begin{abstract}
Purpose] The primary purpose of this study was to evaluate the intraclass correlation coefficient (ICC) in obtaining the cross-sectional area of the lumbar multifidus muscles in patients with chronic low back pain (LBP) at rest and during contractions facilitated by PNF patterns by ultrasound imaging. [Subjects] The subjects were 15 (4 males, 11 females) who had chronic LBP on one side for more than 6 months. [Methods] Subjects were asked to lie on their sides with the painful side facing up. They then rested or received a front or backward lower pelvic pattern of PNF treatment. The cross-sectional area of the multifidus muscle was measured twice using ultrasonography. [Results] The intraclass correlation coefficient of the cross-sectional area of the multifidus muscle measured by ultrasonography was excellent. [Conclusion] Our results show that measurement with ultrasound imaging can be used in the treatment of LBP as an objective assessment.

Key words: Ultrasound imaging, Low back pain, Multifidus muscle
\end{abstract}

(This article was submitted Feb. 18, 2014, and was accepted Apr. 10, 2014)

\section{INTRODUCTION}

Chronic low back pain (LBP) is considered to be a recurring illness. In order to walk upright and maintain an upright posture, a heavy burden is placed on the lumbar region. Many factors leads to LBP, which cannot be managed by a simple treatment.

Chronic LBP is defined as back pain lasting more than 12 weeks, and it affects more than $50 \%$ of the general population. It is estimated that over $70 \%$ of adults have at least one episode of low back pain during their lifetimes. The prevalence of LBP is higher in young, economically active adults; indeed, LBP is the second most common reason for absenteeism from work, and one of the most common reasons for medical consultation. One important risk factor of LBP is weakness of the superficial trunk and abdominal muscles, and strengthening of these muscles is often associated with significant improvements in LBP, as well as with decreased functional disability. Another independent risk factor of LBP is weakness and lack of motor control of the deep trunk muscles, such as the lumbar multifidus and

*Corresponding author. Qiuchen Huang (E-mail: qiuchen_1984@126.com)

(C2014 The Society of Physical Therapy Science. Published by IPEC Inc. This is an open-access article distributed under the terms of the Creative Commons Attribution Non-Commercial No Derivatives (by-ncnd) License $<$ http://creativecommons.org/licenses/by-nc-nd/3.0/>. transverse abdominal muscle ${ }^{1)}$.

Currently, the role of the multifidus muscle in the stabilization of the lumbar region is being given much attention. The inner abdominal muscle thicknesses (multifidus muscle, transverse abdominal muscle) show high correlation with the stability of the lumbar region ${ }^{2}$. Low activity of the inner muscles requires the outer muscles (erector spinae, musculus rectus abdominis, abdominal oblique) to compensate to keep the lumbar region stable. This compensation is one of the causes of LBP.

The lumbar muscle activity of patients with LBP is low compared with healthy subjects ${ }^{3)}$. The multifidus muscle of patients with LBP was reported to be atrophied and replaced by adipose tissue ${ }^{1}$. Furthermore, selective training is necessary for recovery of the multifidus ${ }^{4)}$.

Conservative therapy is used in the treatment of more than $90 \%$ of patients with LBP. "Postural exercise" has been widely used in clinical treatments for the prevention of lumbar lordosis and strengthening of the multifidus muscle. However, because it has not been objectively evaluated, its efficacy is controversial.

Ultrasound imaging has been advocated as a noninvasive method to quantifying muscle morphology and behavior and has been increasingly used both in research and as a clinical tool throughout the rehabilitative process ${ }^{5)}$.

Ultrasound imaging has been validated as a measure of lumbar multifidus muscle morphology through comparisons with magnetic resonance imaging measurements and 
as an indicator of muscle activation with indwelling electromyography ${ }^{6}$. Recently, researchers have been assessing the role of rehabilitative ultrasound imaging in aiding in the evaluation and management of patients with $\mathrm{LBP}^{7}$ ). But most researchers have investigated reliability under limited conditions, most commonly only during resting states repeated during a single testing session. Ultrasound imaging is always used in symptomatic patients for muscles in both resting and contracted states, and the reliability of measurements obtained during exercise or treatment still needs to be examined.

The primary purpose of this study was to evaluate the intraclass correlation coefficient (ICC) in obtaining the crosssectional area of the lumbar multifidus muscles in patients with LBP at rest and during contractions facilitated by PNF patterns the ultrasound imaging.

\section{SUBJECTS AND METHODS}

The subjects were 15 young people ( 4 males, 11 females) who had chronic LBP for more than 6 months on one side. Subjects' characteristics are detailed in Table 1.

The purpose and content of this research were explained to the subjects, and all subjects gave informed consent to participation in the study. The IRB approval number for this study is $12-155$, and the study was approved by the Research Ethics Committee of the International University of Health and Welfare.

Before treatment, the subjects were evaluated for pain severity using a visual analogue scale (VAS). Measurements of the cross-sectional area of the bilateral multifidus muscle were obtained under 3 conditions: in the resting state and during administration of two PNF patterns.

The following two PNF patterns were administered to subjects while they lay on their sides, with the painful side facing up: the front lower pelvic pattern of PNF; and the backward lower pelvic pattern of PNF.

For the front lower pelvic pattern of PNF, the two hands

Table 1. Subject characteristics

\begin{tabular}{lr}
\hline \multicolumn{2}{c}{$\mathrm{M} \pm \mathrm{SD} \mathrm{n}=15$} \\
\hline Age $(\mathrm{y})$ & $19.9 \pm 1.3$ \\
Height $(\mathrm{cm})$ & $161.8 \pm 7.7$ \\
Weight $(\mathrm{kg})$ & $55.1 \pm 7.5$ \\
BMI & $21.1 \pm 2.8$ \\
VAS & $3.3 \pm 1.7$ \\
\hline
\end{tabular}

of the examiner were placed against the upper knee. When subjects performed the front lower pelvic pattern, traction and resistance were added throughout the process. Static resistance and traction were provided in opposition to the PNF pattern by the examiner.

For the backward lower pelvic pattern of PNF, the two hands of the examiner were placed against the upper ossa sedentarium. When subjects performed the front lower pelvic pattern, traction and resistance were added throughout the process. Static resistance and traction were provided in opposition to the PNF pattern by the examiner.

The maximum, contraction which was maintained for 5 seconds, was performed in the middle part of each intervention pattern. At this time, the cross-sectional areas of the bilateral multifidus muscle were measured. Each measurement was made twice.

Ultrasound images of the multifidus muscle wall were obtained using an ultrasound scanner (SSD-650CL, ALOKA, Tokyo, Japan) in the B mode with a $7.5 \mathrm{MHz}$ linear transducer. Gel was applied to the skin beneath the transducer. The transducer was placed on the skin $25 \mathrm{~mm}$ distal from the spinous process of L5 and vertical to the vertebral column. All measurements were carried out by the same physical therapist.

In order to determine the reliability of rehabilitative ultrasound imaging of the cross-sectional area of the lumbar multifidus muscle, the intraclass correlation coefficient (ICC) was used; the factor was the cross-sectional area of the multifidus muscle from two measurements. The data were analyzed using SPSS Ver. 17.0 for Windows.

\section{RESULTS}

The results for the cross-sectional area of the multifidus muscle of the 15 subjects are shown in Table 2 .

All ICCs (1.1) of the multifidus muscle cross-sectional area on both sides were more than 0.93 when the front lower pelvic pattern of PNF and the backward lower pelvic pattern of PNF were carried.

\section{DISCUSSION}

Although the lumbar stabilization exercise is emphasized in clinical practice, there is no objective assessment method for the treatment. In this research, we evaluated the reliability in obtaining cross-sectional area measurements of the lumbar multifidus muscle by ultrasound imaging at rest and during PNF patterns. The reliability of the multifidus muscle cross-sectional area which was measured by

Table 2. The cross-sectional area of the multifidus muscle $\left(\mathrm{cm}^{2}\right)$ and ICC

\begin{tabular}{lcccccc}
\hline & $\begin{array}{c}\text { Pain side in rest } \\
\text { state }\end{array}$ & $\begin{array}{c}\text { Not pain side in } \\
\text { rest state }\end{array}$ & $\begin{array}{c}\text { Pain side in the } \\
\text { front lower pelvic } \\
\text { pattern of PNF }\end{array}$ & $\begin{array}{c}\text { Not pain side in the } \\
\text { front lower pelvic } \\
\text { pattern of PNF }\end{array}$ & $\begin{array}{c}\text { Pain side in the } \\
\text { backward lower } \\
\text { pelvic pattern of PNF }\end{array}$ & $\begin{array}{c}\text { Not pain side in the } \\
\text { backward lower } \\
\text { pelvic pattern of PNF }\end{array}$ \\
\hline 1st time & $7.2 \pm 1.4$ & $8.6 \pm 1.6$ & $10.3 \pm 4.3$ & $9.6 \pm 2.5$ & $8.9 \pm 2.7$ & $10.3 \pm 2.4$ \\
2nd time & $7.2 \pm 1.4$ & $8.6 \pm 1.6$ & $9.6 \pm 3.2$ & $9.4 \pm 2.7$ & $8.9 \pm 2.2$ & $10.2 \pm 2.7$ \\
ICC & $0.99^{* *}$ & $0.99^{* *}$ & $0.93^{* *}$ & $0.98^{* *}$ & $0.98^{* *}$ & $0.97^{* *}$ \\
\hline
\end{tabular}

$* \mathrm{p}<0.05 ; *$ p $<0.01$ 
ultrasound imaging was excellent. Whether we measured the pain side or no-pain side, the constriction of the multifidus muscle could be monitored by ultrasound imaging. So measurements obtained by ultrasound imaging could be used in treatment of LBP as an objective assessment. The effect of conservative treatment for example the postural exercise also could evaluate, compare to past assessment subjectivity method, the ultrasound imaging was intuitional and operable.

In the clinic, the ultrasound imaging could also be used as a real-time measurement, and provide feedback to a therapist that could aid in selection of a more effective treatment method. The ICC could indicate the consistency between the two measurements, but the internal errors could not be expressed. Furthermore, the measurements in this study were carried out by one experimenter on one day, so if these conditions were changed, the reliability of ultrasound imaging would have to be researched again.

Future studies are needed to investigate the inter-rater reliability, standard error of measurement and minimal detectable change of cross-sectional area measurements of the multifidus muscle obtained by ultrasound imaging.

\section{REFERENCES}

1) Hides JA, Richardson CA, Jull GA: Multifidus muscle recovery is not automatic after resolution of acute, first-episode low back pain. Spine, 1996, 21: 2763-2769. [Medline] [CrossRef]

2) Richardson C: Spinal segmental stability. In: Therapeutic Exercise for Spinal Segmental Stabiliationin Low Back Pain, Edinburgh: Churchill Livingstone, 1999, pp 16-26.

3) Isida K: About lumbar stabilization function-comparison between the normal subjects and low back pain. J Hokkaido Phys Therapist, 2000, 19: $15-18$.

4) Mayer TG, Vanharanta H, Gatchel RJ, et al.: Comparison of CT scan muscle measurements and isokinetic trunk strength in postoperative patients. Spine, 1989, 14: 33-36. [Medline] [CrossRef]

5) Teyhen D: Rehabilitative Ultrasound Imaging Symposium San Antonio, TX, May 8-10, 2006. J Orthop Sports Phys Ther, 2006, 36: A1-A3. [Medline] [CrossRef]

6) Hides J, Wilson S, Stanton W, et al.: An MRI investigation into the function of the transversus abdominis muscle during "drawing-in" of the abdominal wall. Spine, 2006, 31: E175-E178. [Medline] [CrossRef]

7) Hodges PW, Pengel LH, Herbert RD, et al.: Measurement of muscle contraction with ultrasound imaging. Muscle Nerve, 2003, 27: 682-692. [Medline] [CrossRef] 